\title{
On the problem of relaxation for radiation-induced optical effects in some ternary chalcogenide glasses
}

\author{
O.I. Shpotyuk ${ }^{1,3}$, M.M. Vakiv ${ }^{1}$, B. Butkiewicz ${ }^{2}$, \\ A.P. Kovalskiy ${ }^{1}$, and R.Ya. Golovchak ${ }^{1,4}$

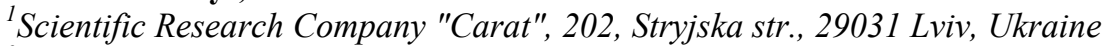 \\ ${ }^{2}$ Institute of Electronic Systems, Warsaw University of Technology, \\ 15/19, Nowowiejska Str., Warsaw 00-665, Poland \\ ${ }^{3}$ Physics Institute, Pedagogical University of Czestochowa, \\ 13/15, Al. Armii Krajowej, Czestochowa 42-415, Poland \\ ${ }^{4}$ Ivan Franko Lviv National University, Physics Department \\ 50, Dragomanov str., 29005 Lviv, Ukraine
}

\begin{abstract}
We have investigated the influence of high-energy $\gamma$-irradiation on the optical transmission spectra of ternary Ge-As-S chalcogenide glasses of the stoichiometric $\mathrm{As}_{2} \mathrm{~S}_{3}-\mathrm{GeS}_{2}$ and non-stoichiometric $\mathrm{As}_{2} \mathrm{~S}_{3}-\mathrm{Ge}_{2} \mathrm{~S}_{3}$ systems. A long-wave shift of the fundamental absorption edge is observed for all glasses. Two different components of radiation-induced changes were specified: static (residual) and dynamic (time dependent) ones. The dynamic component is mathematically described by the exponential and power functions in respect to post-irradiation periods. It is shown that quantitative features of the observed self-restoration phenomena in the irradiated glasses are closely related to their chemical compositions and stoichiometry.
\end{abstract}

Keywords: irradiation, chalcogenide glass, absorption, relaxation.

Manuscript received 07.02.07; accepted for publication 27.09.07; published online 31.10.07.

\section{Introduction}

The great number of chalcogenide glasses (ChGs), thin films, and structures based on these inorganic polymer materials are well known as photosensitive media. The photoinduced changes of optical properties of these amorphous semiconductors have been intensively investigated in recent years. Their microscopic mechanisms as well as physical-chemical features have been studied and explained quite well for the majority of known chalcogenide-based compounds [1]. At the same time, the radiation-induced optical effects (RIOE) produced by high-energy ionizing irradiation ( $\gamma$-quanta, fast electrons, neutrons, etc. with average energies of more than $1 \mathrm{MeV}$ ) have been investigated only for some types of amorphous semiconductors (mainly for binary or quasibinary ones) characterized by a relatively simple two-dimensional structure [2-5]. The systematic investigations of RIOE in more complicated amorphous cross-linked ternary 3D systems obtained in the form of bulk glasses $[6,7]$ have not been carried out yet.
Our interest in such investigations is caused now by different circumstances. The most important is the existence of a topological phase transition at the average coordination number (calculated as the number of covalent chemical bonds per formula unit) $Z \approx 2.67$ in some non-stoichiometric ternary ChGs with the so-called 2D-3D mixed structure. At this point, the compositional dependences of photoinduced effects have wellexpressed peculiarities [7]. As a rule, the extreme values of photoinduced shifts of the fundamental optical absorption edges were observed for $Z \approx 2.67$. The analogous compositional dependences of RIOE in such ChGs with a more complicated mixed 2D-3D structure have not been completely studied yet.

In addition, the time dependent relaxation processes in ChGs treated previously by high-energy ionizing radiation $\left({ }^{60} \mathrm{Co} \gamma\right.$-quanta, for example) need especial attention from the controversial point concerning their degradation or, in other words, the RIOE stability. Despite the widely used conclusion on the full time stability of the radiation-induced changes in ChGs (3-5 years) at room temperature, we revealed that 
these effects measured in the visible range of optical absorption spectra contained two basically different parts: the static (residual) component being constant at room temperature after irradiation and the dynamic (time dependent) one decaying with time at room temperature [8]. But the compositional dependences of these restoration processes, their total duration, and the mathematical description in respect to the post-irradiation exposure are the problems for precise experimental investigations.

Taking into account these features, we put forward the purpose of the detailed study and a mathematical simulation of the restoration processes for $\gamma$-irradiated ternary ChGs of the Ge-As-S system depending on their chemical compositions in a wide range of variation of $Z$ including the point of the $2 \mathrm{D}-3 \mathrm{D}$ topological phase transition.

\section{Experimental procedure}

ChGs of the ternary Ge-As-S system with different average coordination numbers $Z$ are used in the form of disk-like (1 $\mathrm{mm}$ in thickness and $10 \mathrm{~mm}$ in diameter) polished samples of the stoichiometric $\left(\mathrm{As}_{2} \mathrm{~S}_{3}\right)_{\mathrm{y}}\left(\mathrm{GeS}_{2}\right)_{1-\mathrm{y}}$ $(y=0.1, Z=2.63 ; y=0.2, Z=2.59 ; y=0.4, Z=2.52$; $y=0.6, \quad Z=2.48) \quad$ and non-stoichiometric $\left(\mathrm{As}_{2} \mathrm{~S}_{3}\right)_{\mathrm{x}}\left(\mathrm{Ge}_{2} \mathrm{~S}_{3}\right)_{1-\mathrm{x}}(x=0.1, Z=2.76 ; x=0.2, Z=2.72$; $x=0.4, Z=2.64 ; x=0.6, Z=2.56 ; x=0.8, Z=2.48$ ) compositions. All investigated samples were prepared by the melt quenching under natural conditions of a mixture of high purity (99.9999 \%) Ge, As, and S elements. The initial materials were sealed in quartz ampoules at $10^{-3} \mathrm{~Pa}$ and heated gradually up to $1080 \mathrm{~K}$. The furnace was rocked for $24 \mathrm{~h}$ in order to obtain the most homogeneous samples. Then the ingots were quenched in air at ambient temperature. All ampoules were annealed once more at a temperature of $20-30 \mathrm{~K}$ below the softening point $\left(T_{\mathrm{g}}\right)$ to remove the mechanical strains. The amorphous state of the obtained materials was controlled by the existence of a characteristic conchoidal fracture and the data of X-ray diffraction and infrared microscope analyses. Finally, all ingots were sliced into 1-mm thick disks and polished for precise optical measurments.

All samples were irradiated from a ${ }^{60} \mathrm{Co}$ source with a dose of $10^{6}$ Gy and an average energy of $1.25 \mathrm{MeV}$.

Optical investigations were performed in the range of 200-900 nm using a "Specord M40" spectrophotometer. The transmission spectra $\tau(h v)$ at various photons energies $h v$ were measured before and after irradiation in different periods of time (up to 6 months). Then the absorption coefficients $\alpha$ were calculated from the equation [9]

$\alpha=\frac{1}{d} \ln \frac{(1-r)^{2}}{\tau}$,

where $d$ is the sample thickness, and $r$ is the reflectivity.
Table 1. Quantitative parameters of radiation-induced changes of optical absorption for stoichiometric $\left(\mathrm{As}_{2} \mathrm{~S}_{3}\right)_{\mathrm{y}}\left(\mathrm{GeS}_{2}\right)_{1-\mathrm{y}}$ chalcogenide glasses.

\begin{tabular}{|c|c|c|c|c|c|c|}
\hline$y$ & $Z$ & 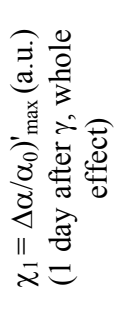 & $\begin{array}{c}h v_{\text {max }}^{\prime} \\
(\mathrm{eV})\end{array}$ & 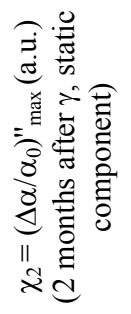 & $\begin{array}{c}h v^{\prime \prime}{ }_{\max } \\
(\mathrm{eV})\end{array}$ & 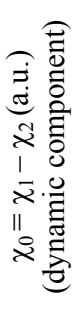 \\
\hline 0.1 & 2.63 & 0.55 & 2.410 & 0.53 & 2.415 & 0.02 \\
\hline 0.2 & 2.59 & 0.52 & 2.380 & 0.48 & 2.390 & 0.04 \\
\hline 0.4 & 2.52 & 0.46 & 2.340 & 0.36 & 2.360 & 0.10 \\
\hline 0.6 & 2.48 & 0.41 & 2.210 & 0.26 & 2.240 & 0.15 \\
\hline
\end{tabular}

Radiation-induced optical changes have been determined by the value of $\chi=\Delta \alpha / \alpha_{0}$, where $\Delta \alpha$ is the difference between the $\alpha$ values before and after $\gamma$ irradiation, and $\alpha_{0}$ is the absorption before $\gamma$-irradiation. To estimate the time dependences of the dynamic component relaxation, the value of $\Delta \alpha / \alpha_{0 \max }$ was taken (the value at the maximum points of $\Delta \alpha / \alpha_{0}(h v)$ dependences).

\section{Results and discussion}

It is well known that $\gamma$-irradiation lead to a long-wave shift of the optical absorption edge of a $\mathrm{ChG}$ due to the specific topological coordination defect formation processes [9]. Some of these defects are unstable with time by causing the existence of the dynamic RIOE component.

The typical spectral $\chi(h v)$ dependences for the RIOE can be presented by characteristic bell-like curves. Such a dependence is shown in Figure for one of the investigated samples $-\left(\mathrm{As}_{2} \mathrm{~S}_{3}\right)_{0.1}\left(\mathrm{Ge}_{2} \mathrm{~S}_{3}\right)_{0.9}$ with $Z=2.76$. Curves 1 and 2 show the change of the $\chi$ parameter with photon energy registered in 1 day and 2 months after irradiation, respectively. The wide maximum, sharp high-energy tail, and wide low-energy one are proper for all spectra. This asymmetry of the $\chi(h v)$ curve becomes sufficiently less with the self-restoration of the gammainduced changes.

One can see that the radiation-stimulated darkening takes place after $\gamma$-treatment. This darkening is unstable and decays with time to some residual value (the static component of RIOE). The relaxation of the dynamic component is considered in this paper in dependence on the stoichiometry of $\mathrm{ChG}$ and the average coordination number $Z$. The quantitative parameters of these static and dynamic RIOE components are presented in Tables 1 and 2 for the investigated stoichiometric and non-stoichiometric ChG systems, respectively. 


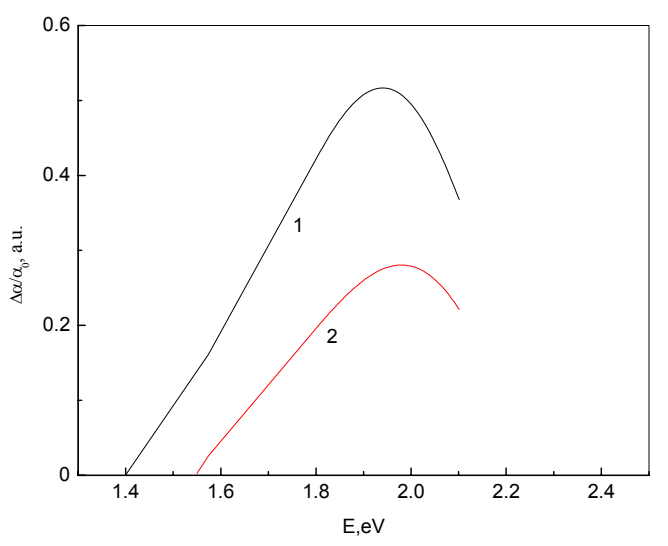

In stoichiometric $\left(\mathrm{As}_{2} \mathrm{~S}_{3}\right)_{\mathrm{y}}\left(\mathrm{GeS}_{2}\right)_{1-\mathrm{y}}$ glasses, the compositional dependence of the dynamic RIOE is determined by the compactness behavior with variation in $Z$. Increasing the $\mathrm{GeS}_{2}$ content in the glass matrix is accompanied by the compactness decrease (owing to the decompressing of the covalent linked network of $\mathrm{As}_{2} \mathrm{~S}_{3}$ based pyramidal units by two structurally inconsistent $\mathrm{GeS}_{2}$ forms) [10]. As a result, the relaxation processes decay too (see Table 1). In the non-stoichiometric $\left(\mathrm{As}_{2} \mathrm{~S}_{3}\right)_{\mathrm{x}}\left(\mathrm{Ge}_{2} \mathrm{~S}_{3}\right)_{1-\mathrm{x}}$ system, this process is complicated by the presence of homopolar chemical bonds with low dissociation energy which enlarge sufficiently the relaxation effects. That is why the compositional dependence of the dynamic RIOE in non-stoichiometric ChGs reveals some peculiarities at the higher $Z$ (see Table 2).

We can see also from Tables 1 and 2 that the spectral position of $\chi_{\max }$ correlates with the change of the band gap $E_{g}$ of the investigated glasses [11].

In order to mathematically describe the observed phenomena, we used the exponential and power functions (see Tables 3 and 4).

Table 2. Quantitative parameters of radiation-induced changes of optical absorption for non-stoichiometric $\left(\mathrm{As}_{2} \mathrm{~S}_{3}\right)_{\mathrm{x}}\left(\mathrm{Ge}_{2} \mathrm{~S}_{3}\right)_{1-\mathrm{x}}$ chalcogenide glasses.

\begin{tabular}{|c|c|c|c|c|c|c|c|}
\hline & $x$ & Z & 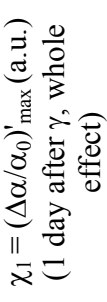 & $\begin{array}{c}h v_{\text {max }}^{\prime} \\
(\mathrm{eV})\end{array}$ & 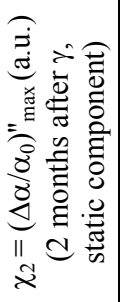 & $\begin{array}{c}h v^{\prime \prime}{ }_{\text {max }} \\
(\mathrm{eV})\end{array}$ & 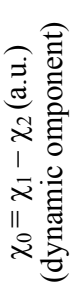 \\
\hline & 0.1 & 2.76 & 0.77 & 1.990 & 0.28 & 2.060 & 0.49 \\
\hline $3 \mathrm{D} \uparrow$ & 0.2 & 2.72 & 0.71 & 2.005 & 0.35 & 2.070 & 0.36 \\
\hline \multicolumn{8}{|c|}{ Topological phase transition } \\
\hline \multirow[t]{3}{*}{$2 \mathrm{D} \downarrow$} & 0.4 & 2.64 & 0.74 & 2.145 & 0.36 & 2.180 & 0.38 \\
\hline & 0.6 & 2.56 & 0.81 & 2.200 & 0.46 & 2.240 & 0.35 \\
\hline & 0.8 & 2.48 & 0.87 & 2.240 & 0.50 & 2.270 & 0.37 \\
\hline
\end{tabular}

Table 3. Parameters of RIOE fitting for stoichiometric $\left(\mathrm{As}_{2} \mathrm{~S}_{3}\right)_{\mathrm{y}}\left(\mathrm{GeS}_{2}\right)_{1-\mathrm{y}}$ glasses.

\begin{tabular}{ccccccc}
\hline$y$ & $Z$ & \multicolumn{2}{c}{$\chi=\chi_{0} \exp \left[-(t / \tau)^{\lambda}\right]$} & \multicolumn{2}{c}{$\chi=\chi_{0} \exp \left(-t / \tau_{1}\right)$} \\
\cline { 3 - 7 } & & $\begin{array}{c}\tau \\
\text { (days) }\end{array}$ & $\begin{array}{c}\lambda \\
\text { (a.u.) }\end{array}$ & Err. & $\tau_{1}$ (days) & Err. \\
\hline 0.1 & 2.63 & 4.75 & 0.06 & 0.00003 & 134.7 & 0.005 \\
0.2 & 2.59 & 3.90 & 0.10 & 0.0003 & 73.1 & 0.019 \\
0.4 & 2.52 & 3.20 & 0.09 & 0.00002 & 88.3 & 0.006 \\
0.6 & 2.48 & 2.65 & 0.14 & 0.0001 & 53.3 & 0.015 \\
\hline
\end{tabular}

Table 4. Parameters of RIOE fitting for non-stoichiometric $\left(\mathrm{As}_{2} \mathrm{~S}_{3}\right)_{\mathbf{x}}\left(\mathrm{Ge}_{2} \mathrm{~S}_{3}\right)_{1-\mathrm{x}}$ glasses.

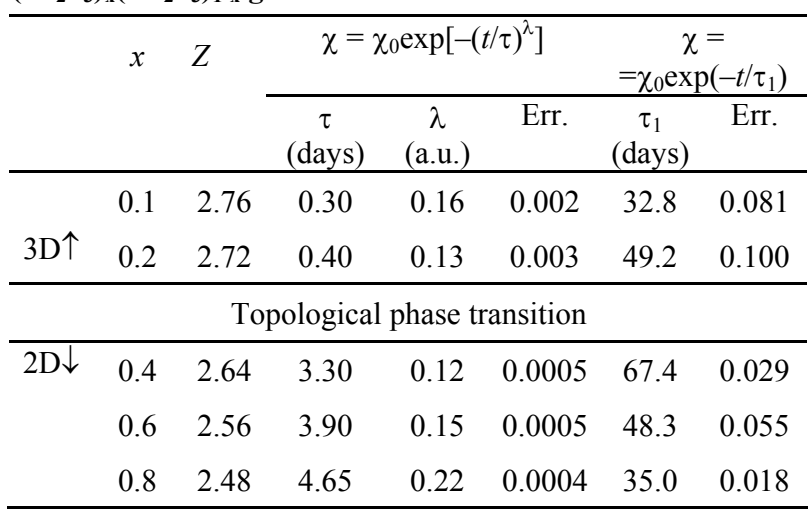

It is worth to note that similar methods of simulation were used previously to describe the temperature dependences of optical reflectivity [12], different types of structural relaxation processes [13-15], and the dose dependences of defect concentrations [16] in various amorphous materials.

The relation

$$
\chi(t)=\chi_{0} \exp \left[-(t / \tau)^{\lambda}\right]
$$

is known as the "universal" relation used to describe the relaxation processes in amorphous solids [16]. The $\chi$ value is the measured quantity, $\tau$ is the time constant and $\lambda$ is the exponent varying from 0 to 1 .

Relation (2) can be considered also as a consequence of the superposition of different relaxation processes [17] and can be replaced by the sum of $n$ exponential functions corresponding to the different types of coordination defects:

$$
\chi(t)=\sum_{i=1}^{n} \chi_{0 i} \exp \left(-t / \tau_{i}\right) .
$$

In the present paper, we make the first attempt to apply expression (2) to the description of RIOE in the investigated ChGs. We also consider separately the case where $\lambda=1$,

$\chi=\chi_{0} \exp (-t / \tau 1)$

and compare the obtained results. 
Calculating the optimum $\tau$ and $\lambda$ values for relation (2), we obtained the results with the lowest error presented in Tables 3 and 4.

The quantitative parameters of mathematical simulation for the dynamic RIOE, $\tau, \lambda$, and $\tau_{1}$, in the stoichiometric $\left(\mathrm{As}_{2} \mathrm{~S}_{3}\right)_{\mathrm{y}}\left(\mathrm{GeS}_{2}\right)_{1-\mathrm{y}}$ system reveal a smooth monotonic dependence on $Z$ (see Table 3 ) without any extrema. While the $\lambda(Z)$ dependence is weakly defined in these ChGs, the compositional $\tau$ and $\tau_{1}$ relations are close to the linear monotonic increase with $Z$. The only exception is the sample with $Z=2.59$, where a high error value was obtained. For the all other investigated samples, the error value is comparatively low. It testifies to the good fitting of experimental data by the chosen expressions. Relation (2) gives a lower error and therefore is more acceptable for the description of dynamic effects in the stoichiometric system. Evidently, this is caused by the complexity of the microstructural mechanism of relaxation. This relaxation process cannot be considered as the single integral process described by the exponential function but as a superposition of several components.

Compositional dependences of the quantitative parameters of the dynamic component differ radically in the non-stoichiometric glasses. They reveal less or more pronounced extrema at the point $Z=2.67$. The quantity $\lambda$ has a local minimum at this point changing the value from 0.12 to 0.22 . On the contrary, $\tau_{1}$ reaches the local maximum changing from 32.8 to 67.4 . The $\tau$ values decrease with $Z$ in the whole range, but, at the point of the topological phase transition, the clear jump of $\tau$ is observed. In 2D compositions, the magnitude of this jump equals several days. But, in 3D compositions, it is less than 1 day. At the same time, the all previous conclusions concerning the effectiveness of the fitting of the obtained experimental data by the chosen mathematical formulas remain actual (see the error values in Table 4). In other words, the fitting by formula (2) of the data concerning the stoichiometric system is better.

Thus, the characteristic feature of the nonstoichiometric glasses in comparison with the stoichiometric ones is the well-pronounced manifesttation of extrema at the point $Z=2.67$. We consider that the specific character of the short- and medium-range order topologies near the point of the topological phase transition in these glasses is the reason for such a behavior. This specific character consists in the anomalously high content of the homopolar chemical bonds which are the "outflows" for the annihilation of radiation-induced coordination defects, by owing to the low dissociation energies. The specific microstructural mechanisms for such an annihilation, as well as the initiating processes of radiation-induced defect formation, need a more detailed study by the methods of structural-physical investigations.

\section{Conclusions}

The relaxation of radiation-induced optical effects in ternary chalcogenide glasses can be described quite well by Eq. (2). This result agrees with the previous data obtained for different types of relaxation in the range of temperatures below $T_{g}$.

The compositional dependences of relaxation parameters in non-stoichiometric ternary glasses, in contradistinction to stoichiometric ones, reveal some peculiarities at the point of the topological phase transition. These features are related to the types of defects in the structure and with the value of glass compactness at different $Z$.

\section{Acknowledgements}

The authors would like to express the thanks to the colleagues from the Institute of Solid State Physics of Bulgarian Academy of Sciences for the collaboration and preparation of samples. This work was supported in part by the Mianowski Foundation (Warsaw, Poland) and the Polish State Committee for Scientific Research.

\section{References}

1. K. Shimakawa, A. Kolobov, S.R. Elliott, Photoinduced effects and metastability in amorphous semiconductors and insulators // Adv. Phys. 44, p. 475 (1995).

2. O.I. Shpotyuk, V.O. Balitska, M.M. Vakiv, Effect of electron-induced dichroism in vitreous $\mathrm{As}_{2} \mathrm{~S}_{3} / /$ J. Non-Cryst. Solids 227-230, p. 837 (1998).

3. V.O. Balitska, O.I. Shpotyuk, Radiation-induced structural transformations in vitreous chalcogenide semiconductors // J. Non-Cryst. Solids 227-230, p. 723 (1998).

4. O.I. Shpotyuk, V.O. Balitska, M.M. Vakiv, L.I. Shpotyuk, Sensors of high-energy radiation based on amorphous chalcogenides // Sensors \& Actuators A - Phys. A 68, p. 356 (1998).

5. O. Shpotyuk, V. Balitska, Coordination defects in vitreous $\mathrm{As}_{2} \mathrm{~S}_{3}$ induced by $\gamma$-irradiation // Acta physica polonica A 92, p. 577 (1997).

6. D. Arsova, E. Vateva, E. Skordeva, V. Petkov, New features of the medium range order in $\mathrm{Ge}_{\mathrm{x}} \mathrm{As}_{40-\mathrm{x}} \mathrm{SSe}_{60}$ glasses // Solid State Communs 98, p. 595 (1996).

7. M. Popescu, F. Sava, A. Lorinczi, E. Skordeva, P-J. Koch, H. Bradaczek, Photo-induced softening and hardening in Ge-As-S amorphous films // $J$. Non-Cryst. Solids 227-230, p. 719 (1998).

8. O.I. Shpotyuk, E.R. Skordeva, R.Ya. Golovchak, V.D. Pamukchieva, A.P. Kovalsky, Radiationstimulated changes of spectral characteristics of the 
optical transmission of chalcogenide glasses $\mathrm{As}_{2} \mathrm{~S}_{3}$ $\mathrm{Ge}_{2} \mathrm{~S}_{3}$ // Zhurnal Prikladnoi Spektroskopii 66, p. 5 (1999) (in Russian).

9. O.I. Shpotyuk, A.O. Matkovsky, A.P. Kovalsky, M.M. Vakiv, Radiation-induced changes of amorphous $\mathrm{As}_{2} \mathrm{~S}_{3}$ physical properties // Radiation Effects \& Defects in Solids 133, p. 1 (1995).

10. E.R. Skordeva, D.D. Arsova, A topological phase transition in ternary chalcogenide films // J. NonCryst. Solids 192\&193 (1995).

11. D. Arsova, Bond arrangment and optical band gap in $\mathrm{Ge}_{\mathrm{x}} \mathrm{As}_{40-\mathrm{x}} \mathrm{SSe}_{60}$ glasses and thin films // J. Phys. Chem. Sol. 57 (1996).
12. L. Boesch, C.T. Moynihan, P.B. Macedo, Conductivity and dielectric relaxation in a potassium silicate glass as a function of thermal history // Amer. Ceram. Soc. Bull. 51, p. 365 (1972).

13. G. Williams and D.C. Watts // Trans. Faraday Society 66, p. 80 (1970).

14. G.P. Johari // Phil. Mag. B 46, p. 549 (1982).

15. T. Egami // Mater. Res. Bull. 13, p. 557 (1978).

16. D.L. Griscom, M.E. Gingerich, E.J. Friebele, Radiation-induced defects in glasses: origin of power-law dependence of concentration on dose // Phys. Rev. Lett. 71, p. 1019 (1993).

17. C.T. Moynihan and P.K. Gupta, // J. Non-Cryst. Solids 29, 143 (1978). 